\title{
Smoking Reduces Conflict-Related Anterior Cingulate Activity in Abstinent Cigarette Smokers Performing a Stroop Task
}

\author{
Allen Azizian ',2,5, Liam J Nestor', Doris Payer', John R Monterosso ${ }^{1,2}$, Arthur L Brody ${ }^{1,3}$ and \\ Edythe D London*, I,3,4
}

'Department of Psychiatry and Biobehavioral Sciences, University of California, Los Angeles, CA, USA; ${ }^{2}$ Department of Psychology, University of Southern California, Los Angeles, CA, USA; ${ }^{3}$ Brain Research Institute, University of California, Los Angeles, CA, USA; ${ }^{4}$ Molecular and Medical

Pharmacology, David Geffen School of Medicine, University of California, Los Angeles, CA, USA

\begin{abstract}
Prior research suggests that abrupt initiation of abstinence from cigarette smoking reduces neural cognitive efficiency. When cognitive efficiency is high, processing speed and accuracy are maximized with minimal allocation of cognitive resources. The study presented here tested the effects of resumption of smoking on cognitive response conflict after overnight abstinence from smoking, hypothesizing that smoking would enhance cognitive efficiency. Twenty paid research volunteers who were chronic cigarette smokers abstained from smoking overnight ( $>12 \mathrm{~h}$ ) before undergoing $\mathrm{fMRI}$ while performing a color-word Stroop task during two separate test sessions: one that did not include smoking before testing and another one that did. Statistical analyses were performed by modeling the Stroop effect (incongruent > congruent) BOLD response within a collection of a priori regions of interest that have consistently been associated with cognitive control. Behavioral assessment alone did not reveal any significant differences in the Stroop effect between the two sessions. BOLD activations, however, indicated that in the right anterior cingulate cortex (ACC), smokers had significantly less task-related activity following smoking $(p<0.02)$. In contrast, the right middle frontal gyrus exhibited significantly greater activity after smoking as compared to the no-smoking session $(p<0.003)$. Exaggerated neural activity in the ACC during nicotine withdrawal may reflect a compensatory mechanism by which cognitive control networks expend excessive energy to support selective attention processes. Resumption of smoking may enhance cognitive control in smokers, involving a reduction in ACC response conflict activity together with improvement in conflict resolution involving the dorsolateral prefrontal cortex.
\end{abstract}

Neuropsychopharmacology (2010) 35, 775-782; doi: I0.1038/npp.2009.I86; published online II November 2009

Keywords: nicotine dependence; nicotine withdrawal; cognitive control; fMRl; Stroop task

\section{INTRODUCTION}

While most cigarette smokers endorse the desire to quit, it is estimated that only $14-49 \%$ will achieve full abstinence after 6 months (Holmes et al, 2004; Hughes et al, 1999; Hurt et al, 1997; Jorenby et al, 1999; Killen et al, 2000), with reported successful abstinence rates after 12 months of roughly $12 \%$ (Breitling et al, 2009). Relapse to smoking in part reflects withdrawal, which includes difficulty concentrating (Heishman, 1999; Newhouse et al, 2004) and other problems with cognitive functioning (Hatsukami et al, 1989; Parrott and Kaye, 1999; Powell et al, 2002; Pritchard et al, 1992; Rusted et al, 2000; Shiffman et al, 1995), all of which

*Correspondence: Dr ED London, UCLA Semel Institute, 740 Westwood Plaza, Room C8-831, Los Angeles, CA 90095, USA,

Tel: + 310825 0606, Fax: + 3108250812 ,

E-mail: elondon@mednet.ucla.edu

${ }^{5}$ Current address: California Department of Mental Health, Coalinga State Hospital

Received II August 2009; revised 7 October 2009; accepted 8 October 2009 can be reversed by the re-initiation of smoking. Previous fMRI research, using a Stroop paradigm, has also demonstrated that even after only a brief period of abstinence (45-60 min), smokers show greater task-related neural activity than after cigarette smoking (Xu et al, 2007). We have also previously examined nicotine-dependent individuals while they performed the N-Back working memory task under both ad libitum smoking $(<1.5 \mathrm{~h}$ abstinence) and overnight abstinence ( $\geqslant 14 \mathrm{~h}$ abstinence) conditions (Xu et al, 2005), observing higher task-related neural activity in the left dorsolateral prefrontal cortex (DLPFC) following abstinence as compared with smoking. These effects associated with abstinence from smoking may reflect compensatory and adaptive neural functioning in order to cope with the effects of nicotine withdrawal on cognitive control, the implications of which may be an increased susceptibility to smoking relapse (Al'Absi et al, 2002; Domier et al, 2007; Snyder et al, 1989).

Effective everyday mental functioning requires a level of cognitive control, whereby cognition is protected from environmental response conflicts. The neural mechanisms 
by which the brain detects cognitive conflict have largely been established in healthy subjects, with consistent involvement of the anterior cingulate cortex (ACC) (Botvinick et al, 2004; Bush et al, 2000; Ridderinkhof et al, 2004). It has been suggested that when conflict is detected in the ACC, a cognitive control system located in the DLPFC is alerted, and subsequently engages in resolving conflict. The ability to monitor one's behavior within a certain environment during acute drug abstinence may be especially important when there is a need to detect conflicting circumstances and resolve them quickly, especially when environmental stimuli might precipitate drug relapse (Garavan and Stout, 2005). Therefore, examining the behavioral and neural responses to environmental response conflict and its resolution during acute abstinence from smoking may elucidate abnormalities in conflict adaptation that contribute to defective cognitive processing in addiction, and which may contribute to smoking relapse.

The present study aimed to extend knowledge on the neural basis of cognitive response conflict and resolution in individuals who suffer from nicotine addiction. Specifically, we aimed to clarify the effect of the resumption of smoking on cognitive response conflict and resolution in abstinent smokers. Performance- and task-related brain activity of research participants, who were regular smokers but abstained from smoking overnight $(>12 \mathrm{~h})$ and performed a color-word Stroop paradigm during fMRI, was assessed during two separate test sessions: one that did not include smoking before testing and another one that did. Following observations of greater task-related neural activity associated with abstinence from smoking as compared with after smoking (Xu et al, 2005; Xu et al, 2007), we hypothesized that smokers would have worse performance and greater conflict-related ACC neural activity when they were nicotine-abstinent, consistent with compromised functional efficiency during cognitive control. We also predicted that following smoking, there would be improvement in performance and conflict resolution, associated with a reduction in ACC activity, concomitant with increased neural functioning in the DLPFC.

\section{MATERIALS AND METHODS}

\section{Participants}

Twenty participants (13 women), 18-55 years of age (mean \pm SE: $37.4 \pm 10.6$ years), who reported smoking $\geqslant 15$ cigarettes per day (mean \pm SE: $19.3 \pm 4.4$ ) for 2 years (mean pack-years \pm SE: $18.2 \pm 16.4$ ), completed the study. Recruited through flyers and newspaper advertisements, volunteers who passed a telephone screening were invited to continue in-person. After receiving a detailed explanation of the study, qualified participants who agreed to continue, provided written informed consent, as approved by the UCLA Institutional Review Board.

During screening, recent smoking was verified by carbon monoxide (CO) levels in expired air of $\geqslant 10 \mathrm{ppm}$ (Microsmokerlyzer; Bedfont Scientific Ltd, Kent, UK) and presence of urinary cotinine (Accutest NicAlert strips; JANT Pharmacal Corporation, Encino, CA, USA). The participants also completed questionnaires covering demographic, medical, psychiatric, and smoking histories. These included the Shipley Institute of Living Scale (Zachary, 1986), Wender Utah Rating Scale (Ward et al, 1993), Beck Depression Inventory (Beck et al, 1996), and Fagerström Test for Nicotine Dependence (Heatherton et al, 1991).

English language fluency and right-handedness, as indicated by a score $>40$ on the Edinburgh Handedness Questionnaire (Oldfield, 1971), were inclusion requirements. English language proficiency was tested using the Verbal Fluency Test (scores $\leqslant 10$ exclusionary) for those participants whose first language was not English, and intelligence was assessed using the Shipley Institute for Living Scale (scores $\leqslant 85$ exclusionary). Other exclusion criteria included current use of any medications that affect cognitive functioning, prior hospitalization for psychiatric illness, and history of head trauma involving loss of consciousness and/or requiring hospitalization. Psychiatric diagnoses were determined using the Structured Clinical Interview for DSM-IV to exclude participants with current psychiatric disorders (other than nicotine dependence). Detailed drug use data were obtained using the Addiction Severity Index (McLellan et al, 1992). Any participant with a significant drug use history was excluded from study participation. Participants who reported light use of marijuana ( $<1$ joint per week) were allowed to participate, but were instructed to avoid marijuana for the $48 \mathrm{~h}$ prior to testing.

Tests of drug abuse were conducted by urine screening for cocaine, methamphetamine, opioids, $\Delta^{9}$-tetrahydrocannabinol, and benzodiazepines. Any positive drug test resulted in exclusion from study participation.

\section{Experimental Design}

Each participant completed two counterbalanced fMRI test sessions (between 1400 and $1700 \mathrm{~h}$ ), each preceded by overnight abstinence from smoking $(>12 \mathrm{~h})$. During each test session, participants underwent fMRI while performing the Stroop Color-Word Interference Task (see below). One session required continued abstinence before fMRI. In the other, participants each smoked two standard cigarettes, which delivered $0.59 \pm 0.06 \mathrm{mg}$ nicotine each $(9.0 \pm 1.0 \mathrm{mg}$ tar, total dose $\sim 1.2 \mathrm{mg}$ nicotine) (Becker et al, 2008) (Quest 1; Vector Tobacco Inc., Durham, NC, USA). Before fMRI, all participants endorsed having maintained overnight smoking abstinence, which was verified by $\mathrm{CO}$ content in exhaled air $(\leqslant 5 \mathrm{ppm})$. In the session that involved smoking, scanning began approximately $15 \mathrm{~min}$ after each participant had smoked the second cigarette.

On scanning days, self-report measures of cigarette craving and nicotine withdrawal were taken three times: (1) at arrival; (2) 10-15 min after completing smoking (immediately before fMRI); and (3) immediately after fMRI (30-35 min after completing smoking). Participants were tested for cigarette craving on the Urge to Smoke (UTS) Scale (Jarvik et al, 2000) and for nicotine withdrawal on the Shiffman/Jarvik Withdrawal Scale (SJWS) (Shiffman and Jarvik, 1976). On the SJWS and UTS, we calculated the mean self-report score (from scores immediately before and immediately after fMRI) in order to assess how participants were feeling during the fMRI session. We also did this for recorded CO levels (ppm) in each participant. 


\section{Stroop Color-Word Interference Task}

Four color words (RED, BLUE, GREEN, and YELLOW) served as the stimuli in congruent (eg, the word RED displayed in red) and incongruent (eg, the word RED displayed in blue) conditions. The stimuli were presented via magnet-compatible VGA goggles (Resonance Technology, Northridge, CA, USA), which have a field view of approximately 20 degrees vertically and 30 degrees horizontally, and display computer images at $800 \times 600$ pixel resolution. Words were presented one at a time at the center of the screen in Helvetica style font, size 72.

The study used a block design with congruent, incongruent, and rest blocks, presented over two runs (counterbalanced across subjects). Each run consisted of eight congruent, eight incongruent, and 15 rest blocks, with 12 trials per block. During congruent/incongruent blocks, subjects identified the font color of each stimulus word. Subjects were instructed to respond, as quickly as possible, by pressing a button using their right hand. Buttons were pressed with the right index, middle, ring, and baby fingers, corresponding to red, blue, green, and yellow, respectively. Responses were registered using a magnet-compatible, four-button response box. Participants were trained on the correct finger positions before the first run of the task. During rest blocks, they viewed a fixation cross at the center of the screen. Before each block, instructions ('Identify the Color' or 'Rest') were presented for a 2-s period. Within a block, each stimulus was presented for $1200 \mathrm{~ms}$, with an inter-stimulus interval of $300 \mathrm{~ms}$. Each task block lasted $18 \mathrm{~s}$, and each rest block lasted $9 \mathrm{~s}$. Each run of the task lasted approximately $7 \mathrm{~min}$. Dependent measures for the task were the mean number of errors committed and the mean reaction time (RT) for the congruent and incongruent conditions. Trials in which RTs were $\leqslant 200 \mathrm{~ms}$ or $\geqslant 1500 \mathrm{~ms}$ were excluded from analyses, as they were likely to indicate distraction or loss of attention $(>50 \%$ of responses below or above these criteria were incorrect). Only RTs for correct responses were included in the analyses. Errors were rare and occurred in $<5 \%$ of overall trials. We calculated the Stroop effect for each subject (incongruent RT-congruent $\mathrm{RT}$ ) as an index of cognitive response conflict.

\section{Analyses of Behavioral and Self-Report Data}

Behavioral analyses for Stroop errors and RT were conducted using a two-condition (congruent and incongruent) $\times$ twosession (no-smoking vs. smoking) linear mixed-models analysis, allowing us to test for an effect of condition, session, and a condition $\times$ session interaction. Between-sessions analyses for the Stroop effect were conducted using a oneway (no-smoking vs. smoking) linear mixed-models analysis.

The self-report measures were analyzed using a twocondition (arrival vs immediately before and after fMRI collapsed) $\times$ two-session (no-smoking $v s$ smoking) linear mixed-models analysis. In observation of significant condition $\times$ session interactions, follow-up planned comparisons were conducted.

\section{Scanning Parameters}

Functional images were acquired with a 3T Siemens Allegra (Erlangen, Germany) head-only MRI scanner. Localizing scans were acquired first to verify the head position and to identify the AC-PC line for the purpose of establishing the acquisition plane. We then acquired a set of T2-weighted, high-resolution, echo-planar anatomical images (26 slices, aligned to AC-PC line, $4 \mathrm{~mm}$ thick $/ 1 \mathrm{~mm}$ skip, pixel $1.56 \mathrm{~mm}^{2}$ ) covering the entire brain volume, to be used for spatial alignment and to help define the location of the BOLD signal. Functional images were acquired using a gradient-echo-planar image (EPI) sequence (TR: $1500 \mathrm{~ms}$; TE: $30 \mathrm{~ms}$, flip angle: 80 degrees; 26 slices; slice thickness $4 \mathrm{~mm}$ with a $1.0-\mathrm{mm}$ inter-slice interval; matrix $64 \times 64$; inplane pixel resolution: $3.12 \mathrm{~mm}^{2}$ ). Two hundred eighty-two entire brain volumes (26 axial slices) were collected during each run of the Stroop task.

\section{fMRI Data Analyses}

Data were pre-processed using FEAT (fMRI Expert Analysis Tool) from the FMRIB Software Library (www.fmrib.ox. ac.uk/fsl). Pre-statistical processing was as follows: motion correction using the FMRIB's Linear Image Registration Tool (MCFLIRT) (Jenkinson and Smith, 2001); non-brain removal using Brain Extraction Tool (BET) (Smith, 2002); spatial smoothing with a 6-mm full-width half-maximum Gaussian kernel; mean-based intensity normalization; and non-linear high-pass temporal filtering (Gaussian-weighted least-squares straight line fit, with sigma $=25.0 \mathrm{~s}$ ). Statistical analysis was performed by modeling the incongruent $>$ congruent contrast (boxcar functions convolved with the hemodynamic response function) as explanatory variables within the context of the general linear model on a voxel-byvoxel basis. $Z$ (Gaussianized T/F) statistical images were thresholded using clusters determined by $Z=2.3$ and corrected cluster significance level of $p=0.05$. Registration to high-resolution structural images of each individual subject was performed using FLIRT (Jenkinson et al, 2002) and all high-resolution structural images were co-registered to standard (Montreal Neurological Institute) space. Higher-level analyses were performed using FLAME (FMRIB's Local Analysis of Mixed Effects) (Beckmann et al, 2003; Woolrich et al, 2004). Z (Gaussianised T/F) statistical images were first thresholded with a mask containing regions of interest predefined a priori. These included the left and right ACC; DLPFC; inferior frontal gyrus (IFG); and posterior cingulate cortex (PCC). These regions were obtained from the Harvard-Oxford cortical structural atlas in FSL and were combined to form the mask. These regions were chosen based on previous findings that the Stroop effect and cognitive control commonly activate these areas (Carter et al, 1998; Durston et al, 2003; Garavan et al, 1999; MacDonald et al, 2000; Milham et al, 2001; Ullsperger and von Cramon, 2004). Using clusters determined by $Z=2.3$ and a corrected cluster significance threshold of $p=0.05$ (Worsley et al, 1992), voxel-by-voxel paired $t$-tests (nosmoking $v s$ smoking sessions) were then performed as part of this higher-level mixed-effects analysis. Although session order (ie, whether smoking or no-smoking scan occurred first) was randomized across subjects, to determine potential session order effects, we performed an additional analysis using the same statistical model using session order as a covariate of no interest. Results from this model did not 
differ from those from the original model; thus, no session order effects were found.

\section{RESULTS}

\section{Self-Report Measures and CO Levels}

Table 1 shows the effects of overnight abstinence and smoking on cigarette craving and nicotine withdrawal during the two sessions. For UTS scores, there was a significant effect of measurement time $(\mathrm{F}=12.2, \mathrm{df}=1,76$, $p<0.01$; at arrival $>$ during scan); session $(\mathrm{F}=4.9, \mathrm{df}=1$, $76, p<0.05$; no-smoking $>$ smoking); and a measurement time $\times$ session interaction $(\mathrm{F}=9.8, \mathrm{df}=1,76, p<0.01)$. Follow-up planned comparisons for the interaction showed significant difference between the no-smoking and smoking sessions for UTS scores during the scan period $(p<0.01)$. On the craving item of the SJWS, there was a significant effect of measurement time $(\mathrm{F}=11.9, \mathrm{df}=1,76, p<0.01$; at arrival $>$ during scan); no significant effect of session $(\mathrm{F}=3.8, \mathrm{df}=1,76, p=0.06)$; but a significant measurement time $\times$ session interaction $(\mathrm{F}=8.4, \mathrm{df}=1,76, p<0.01)$. Planned comparisons demonstrated a significant difference between the no-smoking and smoking sessions for cigarette craving during the scan period $(p<0.01)$. There was only a significant effect of measurement time for psychological symptoms $(\mathrm{F}=5.6, \mathrm{df}=1,76, p<0.05$; at arrival $>$ during scan), with no other main effects or interactions observed for the physical, sedation or appetite items of the SJWS. Finally, for CO levels (ppm), there was a significant effect of measurement time $(\mathrm{F}=47.8, \mathrm{df}=1,76, p<0.001$; at arrival $>$ during scan $)$; session $(\mathrm{F}=50.6, \mathrm{df}=1, \quad 76$, $p<0.001$; no-smoking $>$ smoking); and a measurement time $\times$ session interaction $(\mathrm{F}=50.6, \mathrm{df}=1,76, p<0.001)$. Follow-up planned comparisons for the interaction showed a significant difference between the no-smoking arrival and smoking during scan $(p<0.001)$ and between the smoking arrival and smoking during scan $(p<0.001)$ sessions.

\section{Behavioral Measures}

For mean errors on the Stroop Task (see Figure 1a), there was a significant effect of task condition $(\mathrm{F}=5.0, \mathrm{df}=1,76$, $p<0.05$; incongruent $>$ congruent); no effect of session $(\mathrm{F}=2.9, \mathrm{df}=1,76, p=0.09)$; and no condition $\times$ session interaction $(\mathrm{F}=0.4, \mathrm{df}=1,76, p=0.5)$. For reaction time (see Figure $1 \mathrm{~b}$ ) there was a significant effect of condition $(\mathrm{F}=28.1, \mathrm{df}=1,76, p<0.001$; incongruent $>$ congruent $) ; \mathrm{a}$ significant effect of session $(\mathrm{F}=9.0, \mathrm{df}=1,76, p<0.01$; nosmoking $>$ smoking); but no condition $\times$ session interaction $(\mathrm{F}=0.1, \quad \mathrm{df}=1, \quad 76, \quad p=0.7)$. The Stroop effect (see Figure 1c) showed no effect of session $(\mathrm{F}=1.0, \mathrm{df}=1,38$, $p=0.3)$.

\section{fMRI Measures}

During both the no-smoking and smoking sessions, participants demonstrated robust BOLD activations across a number of regions (see Figure $2 a$ and $b$ ), including the bilateral anterior and posterior cingulated, and inferior frontal and DLPFCs (Brodmann areas 46 and 9). Table 2 shows the results from the small-volume correction analysis when comparing the no-smoking and smoking sessions on activity in the regions mentioned above related to the Stroop effect (inconcongruent $>$ congruent contrast). For this contrast, we identified a cluster of 154 voxels in the ACC (see Figure 3a), with the local maxima of activity located in the right ACC (see Figure $3 \mathrm{~b}$ ) where participants exhibited a significantly greater BOLD response during the no-smoking session as compared to the smoking session $(p<0.02)$. There was also a cluster of 240 voxels in the right middle frontal gyrus (MFG, see Figure $4 a$ and $b$ ) where participants showed a significantly greater BOLD response

Table I Effects of Abstinence and Smoking on Craving and Nicotine Withdrawal

Test session

\begin{tabular}{|c|c|c|c|c|}
\hline & \multirow{2}{*}{\multicolumn{2}{|c|}{ No-smoking }} & & \\
\hline & & & \multicolumn{2}{|c|}{ Smoking } \\
\hline & Arrival time ${ }^{a}$ & During scan ${ }^{\mathrm{b}}$ & Arrival time & During scan \\
\hline \multicolumn{5}{|c|}{ Shiffman/Jarvik Withdrawal Scale } \\
\hline Craving $^{\mathrm{d}}$ & $5.6 \pm 0.3$ & $5.5 \pm 0.3 * *$ & $5.8 \pm 0.3$ & $4.2 \pm 0.3$ \\
\hline Sedation & $2.9 \pm 0.4$ & $3.0 \pm 0.2$ & $2.6 \pm 0.3$ & $2.6 \pm 0.2$ \\
\hline Appetite & $3.9 \pm 0.2$ & $3.8 \pm 0.2$ & $4.3 \pm 0.3$ & $3.9 \pm 0.2$ \\
\hline
\end{tabular}

Data expressed as means and SE.

${ }^{a}$ Assessments at the beginning of each test day $(14: 00-17: 00 \mathrm{~h})$ after overnight abstinence $(\geqslant 12 \mathrm{~h})$.

bMean of two assessments: (I) 10-15 min after smoking (immediately before scan) and (2) immediately after scan (30-35 min after smoking) for SJWS and UTS. 'Significant measurement time $\times$ session interaction $(F=9.8, \mathrm{df}=\mathrm{I}, 76, p<0.0 \mathrm{I})$; ${ }^{*} * \mathrm{f}<0.0 \mathrm{I}$ (follow-up planned comparisons) for a difference between the two smoking sessions during the scan period.

${ }^{d}$ Significant measurement time $\times$ session interaction $(F=8.4, \mathrm{df}=1,76, p<0.0 \mathrm{l})$; *** $p<0.0$ I (follow-up planned comparisons) for a difference between the two smoking sessions during the scan period. 

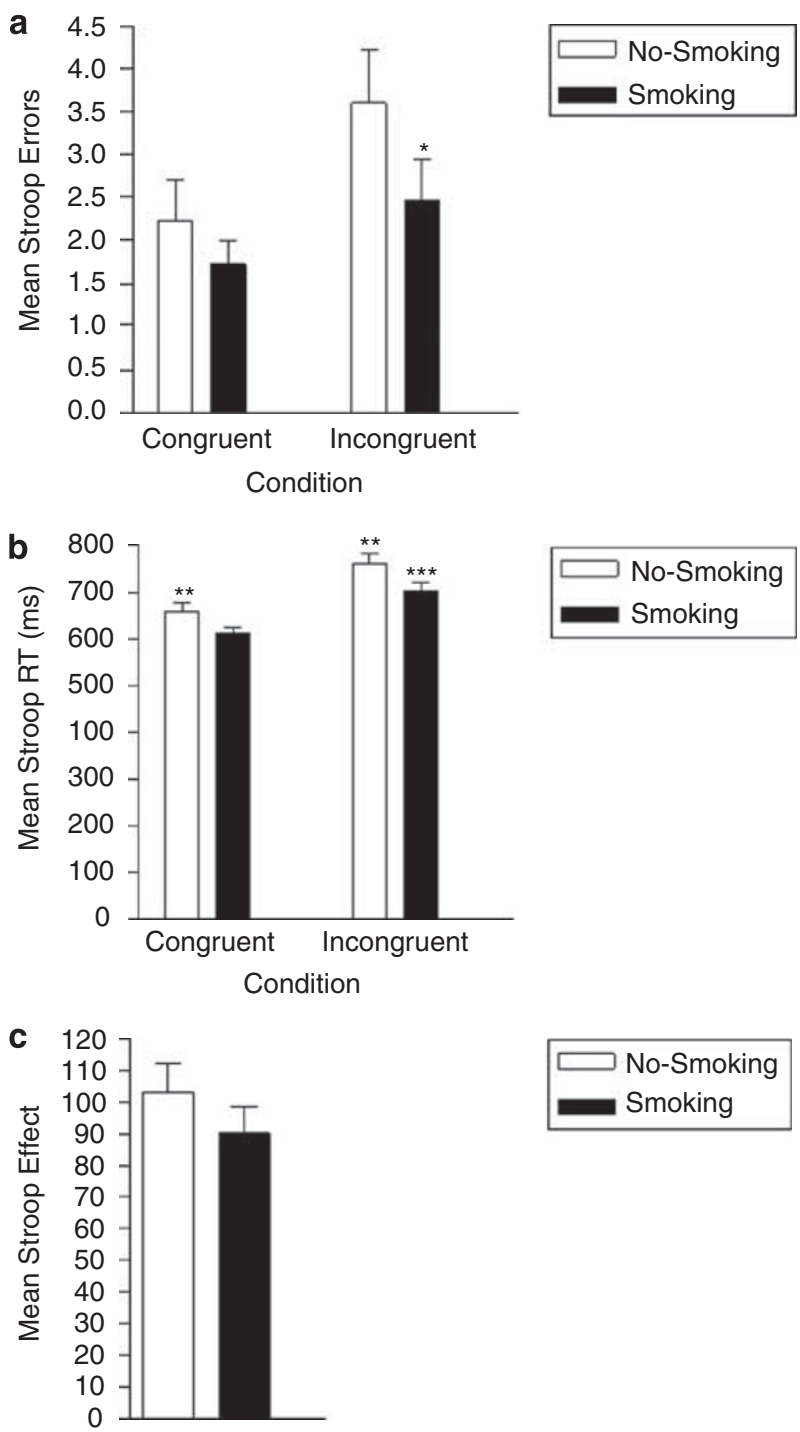

Figure I For the no-smoking and smoking sessions showing: (a) Mean Stroop errors $(* p<0.05$ incongruent $>$ congruent); (b) Stroop reaction time $(* * * p<0.00$ I incongruent $>$ congruent; $* * p<0.0$ I non-smoking $>$ smoking); and (c) mean Stroop effect.

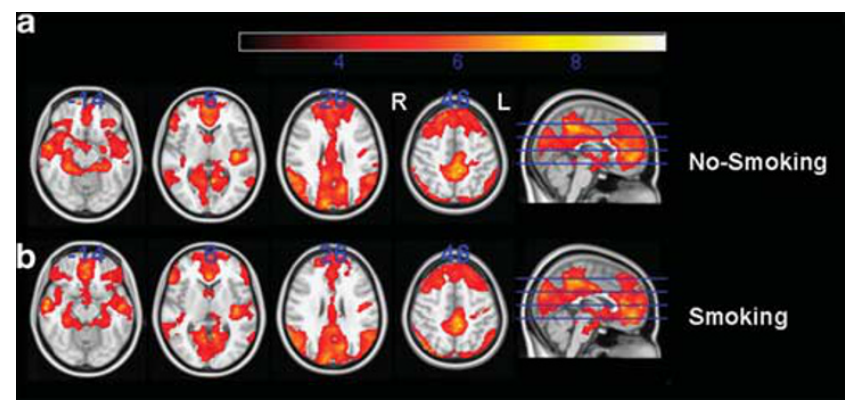

Figure 2 Showing average BOLD activation across the whole brain for the Stroop effect in (a) the no-smoking session and (b) the smoking session. $Z$ (Gaussianized T/F) statistic images were thresholded using clusters determined by $Z=2.3$ and corrected cluster significance level of $p=0.05$. The scale represents the color (from dark to light yellow) of the cluster corresponding to the increasing Z-statistic. The structural image represents the MNII52 average normal brain with corresponding horizontal coordinates (inferior-superior). during the smoking session as compared to the no-smoking session $(p<0.003)$.

\section{Correlations}

There were no correlations between FTND and UTS scores and Stroop performance (ie, errors, reaction time, Stroop effect) in either fMRI test session; nor were there any correlations between nicotine use demographics (ie, packyears, cigarettes per day) and Stroop performance in either fMRI test session.

\section{DISCUSSION}

The study presented here tested the hypothesis that smoking would reduce response conflict and enhance conflict resolution in chronic cigarette smokers who had abstained from smoking for $>12 \mathrm{~h}$. Although we did observe an effect of resumption of smoking on Stroop reaction time, whereby there was a smaller latency to respond in the smoking session, we did not detect any between-session difference in the Stroop effect, which is considered to be an important index of cognitive response conflict. We have previously demonstrated that smoking reduces the Stroop effect in a larger sample of smokers following overnight abstinence (Domier et al, 2007), and there is evidence that nicotine enhances Stroop performance in both smokers and nonsmokers (Provost and Woodward, 1991; Warburton, 1992). Although contradictory findings have been reported concerning this effect (Foulds et al, 1996), the lack of an effect of smoking in abstinent smokers observed here may merely reflect inadequate statistical power given the small sample size.

The neural mechanisms by which the brain detects cognitive conflict have been established, with consistent evidence implicating the ACC in this function (Botvinick et al, 2004; Bush et al, 2000; Ridderinkhof et al, 2004). Our results revealed that for the Stroop effect, there was a significantly smaller BOLD response in the right ACC during the smoking session than during the no-smoking session, suggesting that smoking enhanced cognitive efficiency in abstinent smokers. Cognitive efficiency refers to the allocation of resources toward performance, where it is hypothesized, that under optimal conditions, this allocation is minimized and processing speed and accuracy are maximized (Rypma et al, 2006). While some studies support the view that greater brain activation related to the Stroop effect reflects superior task performance (Bush et al, 1999; Kerns et al, 2005; Strakowski et al, 2005; Zang et al, 2005), other studies suggest that low task-related responses are associated with better cognitive functioning (Kaufmann et al, 2008; Mohanty et al, 2005). These differences are likely related to the use of different versions of the Stroop Task or differential BOLD activation patterns observed between clinical and healthy populations. ACC activity and cognitive conflict are highly correlated, with strong evidence that neural activity within the ACC increases when 'top-down' control is compromised (Botvinick et al, 1999). In the present study, less neural activity in the ACC during the Stroop effect was observed in individuals after smoking following overnight abstinence, perhaps suggesting that reduced neural activity in this region reflected enhanced 
Table 2 Mixed-Effects, Small-Volume Correction Analysis for Incongruent > Congruent Contrast Comparing the Two (No-smoking vs Smoking) Test Sessions

\begin{tabular}{lccccc}
\hline & No. voxels/cluster & $\boldsymbol{x}(\mathbf{m m})$ & $\boldsymbol{y}(\mathbf{m m})$ & $\mathbf{z}(\mathbf{m m})$ & Max Z-statistic \\
\hline $\begin{array}{l}\text { No-smoking }>\text { Smoking } \\
\text { Anterior cingulate cortex }\end{array}$ & 154 & 4 & 22 & 32 & 4.11 \\
$\begin{array}{l}\text { Smoking }>\text { No-smoking } \\
\text { Middle frontal gyrus }\end{array}$ & 240 & 48 & 24 & 28 & 4.17 \\
\hline
\end{tabular}

Statistical images were first thresholded using a mask containing a priori regions of interest, with clusters determined by $Z>2.3$ and a (corrected) cluster significance threshold of $p=0.05$ prior to voxel-by-voxel paired $t$-test analyses. Coordinates represented are in Montreal Neurological Institute (MNI) space. $P$ represents the $P$-value corresponding to the maximum Z-statistic within each cluster. Clusters reported were in the right hemisphere.

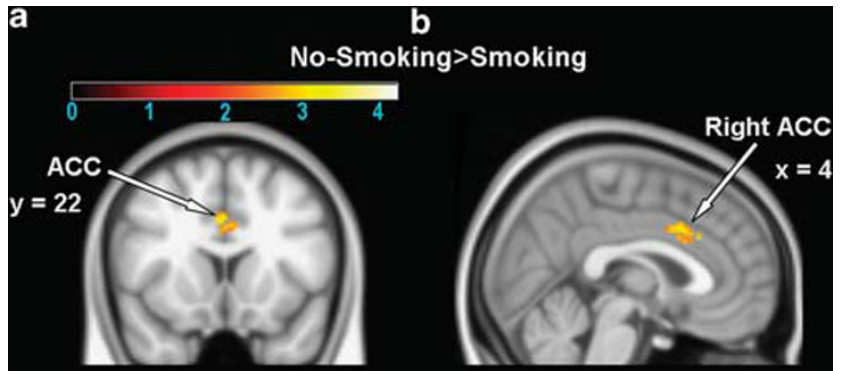

Figure 3 Small-volume correction analysis showing (a) voxel cluster of activity across both the left and right ACC, and (b) the local maxima of activity, located in the right ACC $(x=4, y=22, z=32)$, where smokers showed a significantly greater BOLD response during the no-smoking as compared with the smoking session for the incongruent>congruent contrast ( $p<0.02$, paired $t$-test). The scale represents the color (from dark to light yellow) of the cluster corresponding to the increasing $Z$-statistic. The structural image represents the MNII52 average normal brain with corresponding coronal (anterior-posterior) and sagittal (right-left) coordinates.

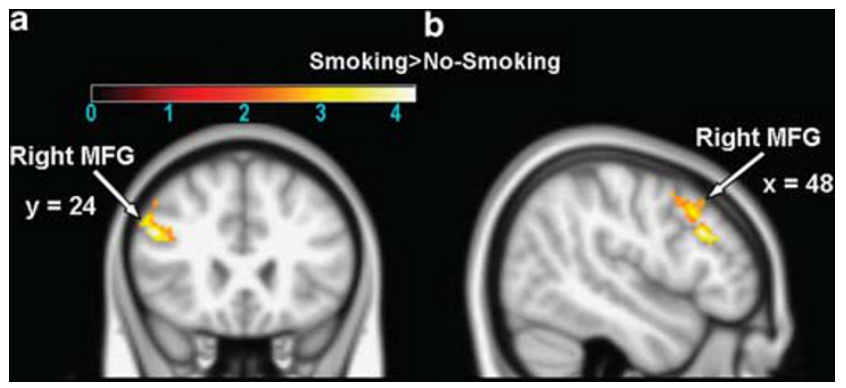

Figure 4 Small-volume correction analysis showing the (a) coronal section and (b) sagittal section voxel cluster of activity located in the right MFG $(x=48, y=24, z=28)$, where smokers showed a significantly greater BOLD response during the smoking as compared with the no-smoking session for the incongruent $>$ congruent contrast $(p<0.003$, paired $t$-test). The scale represents the color (from dark to light yellow) of the cluster corresponding to the increasing $Z$-statistic. The structural image represents the MNII52 average normal brain with corresponding coronal (anteriorposterior) and sagittal (right-left) coordinates.

processing efficiency and reduced response conflict. Exaggerated neural activity during continued abstinence, but not smoking, may therefore, reflect a compensatory mechanism by which cognitive control networks expend excessive energy to support selective attention processes in nicotine addiction. This result of increased neuronal activity appears consistent with an effect indicating possible inefficiency, which we have previously observed in nicotine-dependent subjects performing Stroop and working memory tasks (Xu et al, 2005, 2007).

With respect to conflict adaptation, research suggests that the ACC may be less important than other regions, particularly the DLPFC (Botvinick et al, 1999; Carter et al, 2000). It has been suggested that upon detection of conflict in the ACC, a cognitive control system located in the DLPFC is alerted and subsequently engages in the resolution of conflict. The left DLPFC has previously been associated with the implementation of cognitive control processes in preparation for high-conflict trials (MacDonald et al, 2000), but there is also evidence of conflict adaptation processes in the right DLPFC (Kerns et al, 2004). We observed significantly more neural activity in the right DLPFC (right MFG/BA 9) associated with the Stroop effect following cigarette smoking. This finding appears to be consistent with the current literature, demonstrating increased activity in this region corresponding with high-adjustment, postconflict and post-error trials (Kerns et al, 2004). Computational modeling of the Stroop Task suggests that the DLPFC may be responsible for maintaining and representing context information, which includes the attentional demands of the task (Cohen et al, 1992). Furthermore, cognitive control is implemented in the brain by a distributed network that involves closely interacting, but dissociable, components (MacDonald et al, 2000). The current findings of increased DLPFC activity following smoking, therefore, suggest that re-initiation of smoking re-establishes an optimal dissociation between DLPFC conflict adaptation and ACC response conflict neural activity in smokers.

Interestingly, deficits in conflict monitoring have been observed in a number of clinical populations where cognitive control disturbances have previously been reported. Schizophrenics, for example, exhibit reduced conflictrelated ACC activity (Kerns et al, 2005) as well as reduced conflict adaptation (Kerns et al, 2005) and error-related ACC activation (Alain et al, 2002; Kerns et al, 2005; Mathalon et al, 2002). Research into the neural characteristics of error monitoring in drug-abusing populations has also revealed deficits in ACC functioning (Bolla et al, 2004; Eldreth et al, 2004; Forman et al, 2004; Hester et al, 2009; Kaufman et al, 2003; London et al, 2005), which are contrary to the higher ACC activity observed herein during 
abstinence from smoking. The ACC hyperactivity observed in our abstinent smoking group and hypoactivity reported in schizophrenics and illicit drug users may relate to the use of different cognitive control paradigms, some of which may be more sensitive to the exploits of cognitive conflict monitoring in the ACC.

We have demonstrated important neural activity differences in chronic cigarette smokers, related to nicotine withdrawal and resumption of smoking, during cognitive response conflict and resolution. Specifically, exaggerated neural activity in the ACC during nicotine withdrawal may reflect a compensatory mechanism by which cognitive control networks exhibit a discordant response to support selective attention processes. Moreover, we have shown that resumption of smoking may enhance cognitive control in nicotine addiction, which involves a reduction in ACC response conflict together with improvement in conflict resolution involving the DLPFC. Longitudinal studies, which assess treatment approaches to augmenting nicotine abstinence, may benefit from considering how cognitive control may be compromised during the initial stages of withdrawal, possibly contributing to smoking relapse.

\section{ACKNOWLEDGEMENTS}

This work was supported by NIH grants RO1 DA014093 (EDL) and MOI RR 00865 (UCLA GCRC), UC TobaccoRelated Disease Research Program award 10RT-0091 (EDL), the Philip Morris External Research Program (EDL), an endowment from the Thomas P and Katherine K Pike Chair in Addiction Studies, and a generous gift from the Marjorie M Greene Trust. This paper was presented in part at the annual meeting of the Society for Research on Nicotine and Tobacco in Portland, Oregon, which took place from February 27 to March 1, 2008.

\section{DISCLOSURE}

Dr London received support for other research from Phillip Morris, USA. The other authors have no financial conflicts of interest.

\section{REFERENCES}

Al'Absi M, Amunrud T, Wittmers LE (2002). Psychophysiological effects of nicotine abstinence and behavioral challenges in habitual smokers. Pharmacol Biochem Behav 72: 707-716.

Alain C, McNeely HE, He Y, Christensen BK, West R (2002). Neurophysiological evidence of error-monitoring deficits in patients with schizophrenia. Cereb Cortex 12: 840-846.

Beck A, Steer R, Brown G (1996). Beck Depression InventorySecond Edition manual. The Psychological Corporation: San Antonio, TX.

Becker KM, Rose JE, Albino AP (2008). A randomized trial of nicotine replacement therapy in combination with reducednicotine cigarettes for smoking cessation. Nicotine Tob Res 10: 1139-1148.

Beckmann CF, Jenkinson M, Smith SM (2003). General multilevel linear modeling for group analysis in FMRI. Neuroimage 20: 1052-1063.
Bolla K, Ernst M, Kiehl K, Mouratidis M, Eldreth D, Contoreggi C et al (2004). Prefrontal cortical dysfunction in abstinent cocaine abusers. J Neuropsychiatry Clin Neurosci 16: 456-464.

Botvinick M, Nystrom LE, Fissell K, Carter CS, Cohen JD (1999). Conflict monitoring versus selection-for-action in anterior cingulate cortex. Nature 402: 179-181.

Botvinick MM, Cohen JD, Carter CS (2004). Conflict monitoring and anterior cingulate cortex: an update. Trends Cogn Sci 8: 539-546.

Breitling LP, Twardella D, Raum E, Brenner H (2009). Situational temptation scores and smoking cessation in general care. Psychol Addict Behav 23: 362-367.

Bush G, Frazier JA, Rauch SL, Seidman LJ, Whalen PJ, Jenike MA et al (1999). Anterior cingulate cortex dysfunction in attentiondeficit/hyperactivity disorder revealed by $\mathrm{fMRI}$ and the Counting Stroop. Biol Psychiatry 45: 1542-1552.

Bush G, Luu P, Posner MI (2000). Cognitive and emotional influences in anterior cingulate cortex. Trends Cogn Sci 4: 215-222.

Carter C, Braver T, Barch D, Botvinick M, Noll D, Cohen JD (1998). Anterior cingulate cortex, error detection, and the online monitoring of performance. Science 280: 747-749.

Carter CS, Macdonald AM, Botvinick M, Ross LL, Stenger VA, Noll D et al (2000). Parsing executive processes: strategic vs evaluative functions of the anterior cingulate cortex. Proc Natl Acad Sci USA 97: 1944-1948.

Cohen JD, Servan-Schreiber D, McClelland JL (1992). A parallel distributed processing approach to automaticity. Am J Psychol 105: 239-269.

Domier CP, Monterosso JR, Brody AL, Simon SL, Mendrek A, Olmstead R et al (2007). Effects of cigarette smoking and abstinence on Stroop task performance. Psychopharmacology (Berl) 195: 1-9.

Durston S, Davidson MC, Thomas KM, Worden MS, Tottenham N, Martinez A et al (2003). Parametric manipulation of conflict and response competition using rapid mixed-trial event-related fMRI. Neuroimage 20: 2135-2141.

Eldreth DA, Matochik JA, Cadet JL, Bolla KI (2004). Abnormal brain activity in prefrontal brain regions in abstinent marijuana users. Neuroimage 23: 914-920.

Forman SD, Dougherty GG, Casey BJ, Siegle GJ, Braver TS, Barch $\mathrm{DM}$ et al (2004). Opiate addicts lack error-dependent activation of rostral anterior cingulate. Biol Psychiatry 55: 531-537.

Foulds J, Stapleton J, Swettenham J, Bell N, McSorley K, Russell MA (1996). Cognitive performance effects of subcutaneous nicotine in smokers and never-smokers. Psychopharmacology (Berl) 127: 31-38.

Garavan H, Ross T, Stein E (1999). Right hemispheric dominance of inhibitory control: an eventrelated functional MRI study. Proc Natl Acad Sci USA 96: 8301-8306.

Garavan H, Stout JC (2005). Neurocognitive insights into substance abuse. Trends Cogn Sci 9: 195-201.

Hatsukami D, Fletcher L, Morgan S, Keenan R, Amble P (1989). The effects of varying cigarette deprivation duration on cognitive and performance tasks. J Subst Abuse 1: 407-416.

Heatherton TF, Kozlowski LT, Frecker RC, Fagerstrom KO (1991). The Fagerstrom Test for Nicotine Dependence: a revision of the Fagerstrom Tolerance Questionnaire. Br J Addict 86: 1119-1127.

Heishman SJ (1999). Behavioral and cognitive effects of smoking: relationship to nicotine addiction. Nicotine Tob Res 1(Suppl 2): S143-S147; discussion S165-S146.

Hester R, Nestor L, Garavan H (2009). Impaired error awareness and anterior cingulate cortex hypoactivity in chronic cannabis users. Neuropsychopharmacology 34: 2450-2458.

Holmes S, Zwar N, Jimenez-Ruiz CA, Ryan PJ, Browning D, Bergmann L et al (2004). Bupropion as an aid to smoking cessation: a review of real-life effectiveness. Int J Clin Pract 58: 285-291. 
Hughes JR, Lesmes GR, Hatsukami DK, Richmond RL, Lichtenstein E, Jorenby DE et al (1999). Are higher doses of nicotine replacement more effective for smoking cessation? Nicotine Tob Res 1: 169-174.

Hurt RD, Sachs DP, Glover ED, Offord KP, Johnston JA, Dale LC et al (1997). A comparison of sustained-release bupropion and placebo for smoking cessation. N Engl J Med 337: 1195-1202.

Jarvik ME, Madsen DC, Olmstead RE, Iwamoto-Schaap PN, Elins JL, Benowitz NL (2000). Nicotine blood levels and subjective craving for cigarettes. Pharmacol Biochem Behav 66: 553-558.

Jenkinson M, Bannister P, Brady M, Smith S (2002). Improved optimization for the robust and accurate linear registration and motion correction of brain images. Neuroimage 17: 825-841.

Jenkinson M, Smith S (2001). A global optimisation method for robust affine registration of brain images. Med Image Anal 5: 143-156.

Jorenby DE, Leischow SJ, Nides MA, Rennard SI, Johnston JA, Hughes AR et al (1999). A controlled trial of sustained-release bupropion, a nicotine patch, or both for smoking cessation. $N$ Engl J Med 340: 685-691.

Kaufman JN, Ross TJ, Stein EA, Garavan H (2003). Cingulate hypoactivity in cocaine users during a GO-NOGO task as revealed by event-related functional magnetic resonance imaging. J Neurosci 23: 7839-7843.

Kaufmann L, Ischebeck A, Weiss E, Koppelstaetter F, Siedentopf C, Vogel SE et al (2008). An fMRI study of the numerical Stroop task in individuals with and without minimal cognitive impairment. Cortex 44: 1248-1255.

Kerns JG, Cohen JD, MacDonald III AW, Cho RY, Stenger VA, Carter CS (2004). Anterior cingulate conflict monitoring and adjustments in control. Science 303: 1023-1026.

Kerns JG, Cohen JD, MacDonald III AW, Johnson MK, Stenger VA, Aizenstein $\mathrm{H}$ et al (2005). Decreased conflict- and error-related activity in the anterior cingulate cortex in subjects with schizophrenia. Am J Psychiatry 162: 1833-1839.

Killen JD, Fortmann SP, Schatzberg AF, Hayward C, Sussman L, Rothman $\mathrm{M}$ et al (2000). Nicotine patch and paroxetine for smoking cessation. J Consult Clin Psychol 68: 883-889.

London ED, Berman SM, Voytek B, Simon SL, Mandelkern MA, Monterosso J et al (2005). Cerebral metabolic dysfunction and impaired vigilance in recently abstinent methamphetamine abusers. Biol Psychiatry 58: 770-778.

MacDonald A, Cohen J, Stenger V, Carter C (2000). Dissociating the role of the dorsolateral prefrontal and anterior cingulate cortex in cognitive control. Science 288: 1835-1838.

Mathalon DH, Fedor M, Faustman WO, Gray M, Askari N, Ford JM (2002). Response-monitoring dysfunction in schizophrenia: an event-related brain potential study. J Abnorm Psychol 111: 22-41.

McLellan AT, Kushner H, Metzger D, Peters R, Smith I, Grissom G et al (1992). The fifth edition of the Addiction Severity Index. J Subst Abuse Treat 9: 199-213.

Milham MP, Banich MT, Webb A, Barad V, Cohen NJ, Wszalek T et al (2001). The relative involvement of anterior cingulate and prefrontal cortex in attentional control depends on nature of conflict. Brain Res Cogn Brain Res 12: 467-473.

Mohanty A, Herrington JD, Koven NS, Fisher JE, Wenzel EA, Webb AG et al (2005). Neural mechanisms of affective interference in schizotypy. J Abnorm Psychol 114: 16-27.

Newhouse PA, Potter A, Singh A (2004). Effects of nicotinic stimulation on cognitive performance. Curr Opin Pharmacol 4: 36-46.

Oldfield R (1971). The assessment and analysis of handedness: the Edinburgh inventory. Neuropsychologia 9: 97-113.
Parrott AC, Kaye FJ (1999). Daily uplifts, hassles, stresses and cognitive failures: in cigarette smokers, abstaining smokers, and non-smokers. Behav Pharmacol 10: 639-646.

Powell J, Dawkins L, Davis RE (2002). Smoking, reward responsiveness, and response inhibition: tests of an incentive motivational model. Biol Psychiatry 51: 151-163.

Pritchard WS, Robinson JH, Guy TD (1992). Enhancement of continuous performance task reaction time by smoking in nondeprived smokers. Psychopharmacology (Berl) 108: 437-442.

Provost SC, Woodward R (1991). Effects of nicotine gum on repeated administration of the Stroop test. Psychopharmacology (Berl) 104: 536-540.

Ridderinkhof KR, Ullsperger M, Crone EA, Nieuwenhuis S (2004). The role of the medial frontal cortex in cognitive control. Science 306: 443-447.

Rusted JM, Caulfield D, King L, Goode A (2000). Moving out of the laboratory: does nicotine improve everyday attention? Behav Pharmacol 11: 621-629.

Rypma B, Berger JS, Prabhakaran V, Bly BM, Kimberg DY, Biswal BB et al (2006). Neural correlates of cognitive efficiency. Neuroimage 33: 969-979.

Shiffman S, Jarvik M (1976). Smoking withdrawal symptoms in two weeks of abstinence. Psychopharmacology (Berl) 50: 35-39.

Shiffman S, Paty JA, Gnys M, Kassel JD, Elash C (1995). Nicotine withdrawal in chippers and regular smokers: subjective and cognitive effects. Health Psychol 14: 301-309.

Smith SM (2002). Fast robust automated brain extraction. Hum Brain Mapp 17: 143-155.

Snyder FR, Davis FC, Henningfield JE (1989). The tobacco withdrawal syndrome: performance decrements assessed on a computerized test battery. Drug Alcohol Depend 23: 259-266.

Strakowski SM, Adler CM, Holland SK, Mills NP, DelBello MP, Eliassen JC (2005). Abnormal FMRI brain activation in euthymic bipolar disorder patients during a counting Stroop interference task. Am J Psychiatry 162: 1697-1705.

Ullsperger M, von Cramon DY (2004). Neuroimaging of performance monitoring: error detection and beyond. Cortex 40: 593-604.

Warburton DM (1992). Nicotine as a cognitive enhancer. Prog Neuropsychopharmacol Biol Psychiatry 16: 181-191.

Ward MF, Wender PH, Reimherr FW (1993). The Wender Utah Rating Scale: an aid in the retrospective diagnosis of childhood attention deficit hyperactivity disorder. Am J Psychiatry 150: 885-890.

Woolrich MW, Behrens TE, Beckmann CF, Jenkinson M, Smith SM (2004). Multilevel linear modelling for FMRI group analysis using Bayesian inference. Neuroimage 21: 1732-1747.

Worsley KJ, Evans AC, Marrett S, Neelin P (1992). A threedimensional statistical analysis for CBF activation studies in human brain. J Cereb Blood Flow Metab 12: 900-918.

Xu J, Mendrek A, Cohen MS, Monterosso J, Rodriguez P, Simon SL et al (2005). Brain activity in cigarette smokers performing a working memory task: effect of smoking abstinence. Biol Psychiatry 58: 143-150.

$\mathrm{Xu}$ J, Mendrek A, Cohen MS, Monterosso J, Simon S, Jarvik M et al (2007). Effect of cigarette smoking on prefrontal cortical function in nondeprived smokers performing the Stroop Task. Neuropsychopharmacology 32: 1421-1428.

Zachary R (1986). Shipley Institute of Living Scale: Revised manual. Western Psychological Services: Los Angeles.

Zang YF, Jin Z, Weng XC, Zhang L, Zeng YW, Yang L et al (2005). Functional MRI in attention-deficit hyperactivity disorder: evidence for hypofrontality. Brain Dev 27: 544-550. 